Meta

Journal des traducteurs

Translators' Journal

\title{
La traduction raisonnée
}

\section{Jean Darbelnet}

Volume 14, numéro 3, septembre 1969

URI : https://id.erudit.org/iderudit/002482ar

DOI : https://doi.org/10.7202/002482ar

Aller au sommaire du numéro

Éditeur(s)

Les Presses de l'Université de Montréal

ISSN

0026-0452 (imprimé)

1492-1421 (numérique)

Découvrir la revue

Citer cet article

Darbelnet, J. (1969). La traduction raisonnée. Meta, 14(3), 135-140.

https://doi.org/10.7202/002482ar

Ce document est protégé par la loi sur le droit d'auteur. L'utilisation des services d'Érudit (y compris la reproduction) est assujettie à sa politique d'utilisation que vous pouvez consulter en ligne.

https://apropos.erudit.org/fr/usagers/politique-dutilisation/
Cet article est diffusé et préservé par Érudit.

Érudit est un consortium interuniversitaire sans but lucratif composé de l'Université de Montréal, l'Université Laval et l'Université du Québec à Montréal. Il a pour mission la promotion et la valorisation de la recherche. https://www.erudit.org/fr/ 


\section{LA \\ TRADUCTION RAISONNÉE}

C'est le titre qu'on a donné à deux exposés faits devant la Société des traducteurs du Québec au cours des deux séances d'étude du mois de novembre 1968. On aurait pu également les intituler: "La linguistique différentielle au service de la traduction $»$.

Il y a déjà quelque temps, on le sait, que s'organise, au sein de la linguistique, une discipline que l'on pourrait appeler la linguistique différentielle parce qu'elle s'attache à dégager les différences caractéristiques de deux langues données. Le présent article se propose d'examiner en quoi cette discipline peut contribuer à la formation du traducteur.

Auparavant il convient de rappeler qu'il y a eu d'excellentes traductions avant que s'institue une comparaison systématique de la langue de départ et de la langue d'arrivée et que cette comparaison devienne matière d'enseignement. Mais il en est de la traduction comme de beaucoup d'autres activités intellectuelles où la réussite tient à la fois aux dispositions innées du sujet et au métier qu'il a acquis, et les proportions de l'inné et de l'acquis varient avec les individus. En d'autres termes, on peut et on doit admettre qu'il existe des traducteurs capables de résoudre des problèmes de traduction sans se reporter délibérément à un ensemble de principes. Mais nous sommes à une époque où la demande, dans le domaine de la traduction, dépasse l'offre et où la nécessité se fait de plus en plus sentir de former les traducteurs plutôt que de compter sur leur compréhension intuitive des langues qu'ils utilisent. C'est dans cette perspective qu'on peut légitimement tenter de définir l'apport de la linguistique différentielle à la pratique de la traduction. Nous n'essaierons pas de dire si la traduction est un art ou une science. Elle participe sans doute de l'un et de l'autre. Elle est un art lorsque l'équivalent proposé résulte d'une décision personnelle et audacieuse du traducteur, prise en dehors des sentiers battus. Il y a ainsi des trouvailles de génie. Mais il faut reconnaitre que la plupart des faiblesses que l'on relève dans les traductions se situent à un niveau moins élevé, celui où l'absence de métier s'ajoute à celle du génie. 
Nos lecteurs ne sont pas sans savoir ce qu'est la stylistique comparé, et certains la pratiquent. Ils peuvent donc se demander si, dans ce qui précède, il n'y a pas eu substitution de termes. En fait, la linguistique différentielle comprend la stylistique comparée. Dans les perspectives où nous nous plaçons, la stylistique comparée s'impose à notre attention comme étant la plus utile au traducteur, mais la comparaison de deux langues touche aussi à la grammaire et au vocabulaire. Cette opposition de termes correspond donc à une division du travail. À la stylistique revient tout ce qui est expressif, qu'il s'agisse de syntaxe, de vocabulaire ou même d'accentuation; à la lexicologie, tout ce qui est sens et emploi des mots en dehors de leurs valeurs expressives; de la grammaire relèvent les faits de structure du moment qu'ils sont dénués d'expressivité. Quelques exemples permettront d'éclairer ces distinctions. Ils seront empruntés indifféremment aux trois domaines mentionnés ci-dessus.

Commençons par la lexicologie différentielle qui est le domaine le plus facile à délimiter, les mots étant ce qu'il y a de plus concret. Les FAUX AMIs (deceptive cognates) en occupent la majeure partie. L'étude en a été entreprise il y a quarante ans par Koessler et Derocquigny, mais il reste beaucoup de différences à définir et à classer entre les mots français et anglais de même origine mais de comportement différent. Les notions de sens propre ou figuré, de sens intellectuel ou affectif, d'inhérence et de relation, que nous retrouverons plus loin, permettent de poursuivre cette étude avec plus de précision.

La distinction entre grammaire et stylistique n'est pas évidente à première vue. Elle apparaîtra cependant au moyen de l'exemple suivant. L'anglais ne peut pas donner à l'infinitif actif la valeur d'un impératif. C'est pourquoi ne pas plier se rend par do not fold. C'est là une différence d'ordre structural entre les deux langues, qui n'a rien à voir avec l'expressivité ou les niveaux de langue. Nous restons donc dans le domaine de la grammaire. Mais le français, lui, a le choix entre l'impératif et l'infinitif, et ce choix est d'ordre stylistique. Comparez n'entrez pas!, qui est dramatique, avec défense d'entrer et, dans une rue, sens unique ou sens interdit, qui sont des avis de la langue administrative. On voit comment l'on passe de la grammaire à la stylistique lorsque, la langue disposant de deux tournures, celles-ci sont inégalement expressives ou appartiennent à des niveaux de langue différents.

C'est également la stylistique qui relève les différences de vocabulaire pour marquer l'intensité: strictly prohibited se rend par formellement interdit. Sans doute «strictement prohibé » est-il concevable, mais ce syntagme est en dehors de l'usage, il n'est pas idiomatique. Bien entendu, ce qui vaut pour l'intensité s'applique également à l'expression des autres mouvements de notre sensibilité.

Il est en outre naturel et commode de considérer comme relevant de la stylistique l'étude des mots et des tours qui sont liés à des situations ou à des faits de culture. C'est la situation qui nous permet d'affirmer que la séance est ouverte est bien l'équivalent de the meeting will come to order, et c'est une différence de culture qui fait qu'un lecteur français ignorant la topographie sociale de beaucoup 
de villes nord-américaines se tromperait sur le sens de cette phrase en apparence transparente: He lives on the wrong side of the tracks. Nous dirons que l'identité de situation crée des ÉQUIVALENCES, tandis que les différences de culture entraînent des ADAPTATIONS.

Ce qu'on vient de lire n'est pas vraiment nouveau pour quiconque utilise le manuel de Stylistique comparée du français et de l'anglais. Mais beaucoup de ceux qui s'en servent s'attachent plutôt aux exemples qu'aux concepts. Or, si l'abondance des exemples présente une valeur pratique indéniable, il n'en reste pas moins qu'on se prive d'une aide importante en négligeant les concepts. Sans doute ceux-ci sont-ils par nature des abstractions, mais ils s'attachent à une réalité concrète et en fait ils n'existent que pour montrer ce qu'ont de commun des emplois de la langue courante. Il vaut la peine de le montrer en utilisant des notions dont la plupart sont dans le manuel.

La distinction qu'il convient de faire, par exemple, entre le GramMATrCaL et l'IDIOMATIQue n'est pas une vue de l'esprit, elle se justifie par sa commodité; elle permet en effet de caractériser et de classer certains écarts. Nous appelons « écarts » des déviations par rapport à l'usage reçu. Ainsi l'énoncé le nom est Paquette utilise une construction parfaitement française du point de vue grammatical, mais, pour une raison mystérieuse, elle n'est pas plus idiomatique que 《strictement prohibé » que nous avons vu plus haut. Là où l'anglais n'exerce aucune influence, on dira: Je suis Pierre Paquette ou je m'appelle Paquette. Les limites de l'usage, qui séparent ce qui se dit de ce qui ne se dit pas, sont, on le voit, arbitraires.

L'un des concepts de base de la stylistique comparée est celui des UNITÉS DE TRADUCTION ou unités de pensée. Il n'existe malheureusement pas toujours des critères externes de ces unités, de sorte que le traducteur doit le plus souvent les connaître avant de pouvoir les reconnaître, ou, si l'on préfère, il reconnaît ce qu'il sait déjà. Il y a d'ailleurs des ambiguïtés que le contexte ou l'intonation permettent de dissiper. You are telling me suivi d'une subordonnée représente trois unités, tandis que you are telling me, avec l'accent sur me n'en représente qu'une; les quatre mots ont alors le sens global de je vous crois, je suis payé pour le savoir, vous ne m'apprenez rien. L'unité de pensée s'identifie non seulement par son contour mais par son sens global, par ce qu'il est commode d'appeler son terme d'identification. Ainsi there is no such thing as perfection est un énoncé qui comprend deux unités de traduction, l'une de six mots et l'autre d'un seul, le dernier. Les six premiers mots expriment la négation de l'existence: la perfection n'existe pas, n'est pas de ce monde. Cette expression a un paronyme dans there's nothing like, qui constitue aussi une unité, mais malgré la similitude de structure et de mots, le sens en est très différent; il s'agit d'un sentiment d'admiration qui met la chose admirée plus haut que tout; par exemple, il n'y a rien de tel que la promptitude. Remarquons la similitude de forme entre there's no such thing as..., il n'y a rien de tel que... et there's nothing like... Une analyse structurale qui nierait le sens global de ces 
expressions pourrait fort bien aboutir à mettre en équation la première et la deuxième, alors qu'en fait celle-ci est l'équivalent de la troisième. Nous avons là un bon exemple du danger que présentent les similitudes de forme: il faut se garder de croire qu'elles correspondent toujours à des similitudes de sens.

La classification des sens en SENS PROPRE et FIGURÉ, SENS INTELLECTUEL et AFFECTIF, INHÉRENCE et RELATION (cette dernière catégorie ne concernant que les adjectifs) relève de la sémantique mais touche à la stylistique par le caractère affectif de certains des mots qu'elle catalogue. Ici encore il ne s'agit pas d'abstractions gratuites, mais de moyens de cerner le réel. De plus, ces distinctions peuvent aboutir à un découpage différent du texte à traduire, donc à une autre répartition des mots du texte en unités de traduction.

Les mots grand et film sont associés différemment suivant que je dis: C'est un grand film (a great picture) ou à quelle heure passe le grand film? (the feature). On peut évidemment retenir cette distinction une fois pour toutes sans aller plus loin, mais on pénètre plus avant dans la compréhension des deux langues si l'on reconnaît que dans le premier cas grand a un sens affectif et dans le deuxième un sens intellectuel. On remarquera de plus que le grand film (avec l'article défini) constitue une unité de traduction, tandis que dans un grand film, au sens admiratif, grand et film constituent deux unités distinctes. Nous retenons donc qu'un même adjectif ne se rend pas nécessairement par le même équivalent au sens intellectuel et au sens affectif et de semblables divergences existent entre le sens propre et le sens figuré, l'inhérence et la relation. Ces trois catégories se recouvrent assez souvent, et nous allons en voir des exemples, mais on perdrait à les ramener à une seule à cause des exceptions et aussi de l'éclairage différent qu'elles fournissent sur les mots, même quand elles coïncident. Un cours magistral peut être, suivant l'accent qu'on place sur l'adjectif, soit un cours ex cathedra soit un cours d'une qualité remarquable. Ce que le français marque par des moyens prosodiques, l'anglais l'exprime ici par des moyens lexicaux, c'est-à-dire en changeant de mots: a lecture course, a masterly course. Le terme PROSODIOUE est technique; il se justifie parce qu'il permet de reconnaitre une catégorie de signes qui peuvent être d'ordre sémantique ou stylistique. Dans l'exemple ci-dessus, magistral a soit un sens intellectuel et il marque alors la relation, soit un sens affectif et il est alors adjectif d'inhérence. On pourrait également considérer que son sens intellectuel est en même temps son sens propre, tandis que son sens affectif est son sens figuré. Il arrive souvent que l'adjectif de relation soit rendu en anglais par un substantif qui fonctionne comme adjectif. C'est le cas de lecture dans lecture course. L'adjectif de relation marque une relation plutôt qu'une qualité inhérente à l'objet. Comme tel il ne peut se mettre ni au comparatif ni au superlatif, il n'est jamais antéposé et il ne peut être attribut. Dans ce cours est magistral, nous parlons nécessairement de la qualité du cours et non de la façon dont il est donné.

Il peut arriver que les deux langues utilisent des adjectifs pour l'inhérence et la relation. Dans une voix musicale et une soirée musicale, les deux catégories sont 
représentées. Mais l'usage ne nous permet pas de dire «un instrument musical » pour un instrument de musique. Ici le français a recours à une locution adjectivale pour marquer la relation de l'instrument et de la musique. En anglais musical s'applique indifféremment à la voix (inhérence), à la soirée (relation), et à l'instrument (relation).

De semblable façon, angulaire exprime la relation et anguleux l'inhérence. Les deux adjectifs n'ont qu'une forme en anglais: angular.

La double dichotomie entre le propre et le figuré, l'intellectuel et l'affectif vaut aussi pour les noms et les verbes. Électrifier s'emploie au sens propre et électriser le plus souvent au sens figuré. L'anglais ne possède qu'une forme pour ces deux emplois: to electrify. Par contre, notre verbe patauger possède un sens propre (les enfants pataugent), un sens figuré (laissons-le patauger) et un sens intermédiaire (patauger dans la boue). $\hat{A}$ chacun de ces sens correspond en anglais un verbe différent: to paddle, to flounder, to slosh around.

La grammaire différentielle peut difficilement se passer du concept d'ASPECT pour expliquer certaines différences entre le français et l'anglais. On sait que l'aspect est le stade où se trouve une action au moment où on la considère. L'action peut être à son début, à sa fin, ou envisagée dans sa durée ou sa répétition. Les deux langues connaissent l'aspect mais ne le marquent pas de la même façon. L'anglais utilise surtout ceux de ses adverbes qui peuvent aussi fonctionner comme prépositions: up, down, out, away, etc. Il arrive que le français ait recours à un préfixe; ainsi s'envoler s'oppose à voler. Mais le plus souvent l'aspect est implicite en français, alors qu'il est explicité en anglais. C'est ainsi que usé est rendu, suivant le degré d'usure, par worn away ou worn out, que suspendre, accrocher se rendent par to hang up, coudre un bouton, par to sew on a button, fondre l'argenterie de famille, par to melt down the family's silver, etc. Ces remarques valent surtout pour la traduction du français en anglais.

Tout aussi utile est la distinction entre verbes de MOUvement et verbes de DÉPLACEMENT faite par Lucien Tesnière. Elle permet d'expliquer pourquoi marcher au bureau est un anglicisme. La différence entre le mouvement et le déplacement est que le mouvement n'entraîne pas nécessairement le changement de lieu. En anglais la distinction entre verbes de mouvement et verbes de déplacement est sans objet parce que c'est la préposition ou l'adverbe qui indique s'il y a mouvement à l'intérieur du lieu ou changement de lieu. Comparez: I walk in the park/to the park. En français les prépositions ne sont pas aptes à faire ce travail, qui incombe aux verbes. Or, marcher est un verbe de mouvement et aller ( $\grave{a}$ pied) un verbe de déplacement. D'où la nécessité de dire: Je me rends à pied au bureau. C'est pour la même raison qu'il faut dire: L'indemnité journalière a été portée à vingt dollars, et non augmentée à vingt dollars, car le changement de prix ne peut être marqué que par un verbe de déplacement. Or, augmenter est un verbe de mouvement. 
Il a été question plus haut des moyens prosodiques et, dans l'exemple donné, ces moyens étaient utilisés par le français. En fait c'est le plus souvent l'anglais qui s'en sert, du fait que l'accent de phrase peut y tomber sur n'importe quel mot, ce qui n'est pas possible en français. À propos de la démission du général de Gaulle, la Gazette de Montréal cite le Premier Ministre comme ayant dit: «Beginning tomorrow a new page in our history will be turned. » Et la Gazette ajoute: "It is a new page in the world"s history. " On ne peut lire cette dernière phrase à haute voix sans mettre l'accent sur world's pour dégager l'opposition entre l'histoire de France et l'histoire du monde. En français il est évidemment possible de détacher le mot monde, mais cette mise en relief ne portera que si elle peut s'appuyer sur un autre moyen, à savoir l'adjonction de aussi: "C'est aussi une page de l'histoire du monde. »

Les différences que nous venons d'étudier entre les procédés qu'utilisent les deux langues pour exprimer les mêmes idées montrent indirectement combien est injustifié le scrupule des traducteurs qui croient qu'il faut coller au texte pour être sûr de ne pas se tromper. On a pu voir par les exemples qui se sont succédé à propos des concepts examinés, que la bonne traduction est celle qui rend le sens de la langue de départ avec les moyens de la langue d'arrivée. Vérité évidente, dira-t-on. Sans doute, mais nombreux sont encore les traducteurs qui subissent la tyrannie de la forme. C'est en pensant à eux qu'Hilaire Belloc a dit: "The translator must be emancipated from mechanical restriction, of which the two chief forms are the restriction of space and the restriction of form. 》

JeAn Darbelnet 